\title{
Myxoedema Coma - importance of early recognition!
}

\author{
R Wadhwani ${ }^{1}$ S Mashayekhi ${ }^{1}$, A Sharma ${ }^{1}$, Prof S Robinson ${ }^{1}$
}

Endocrinology Department, St Mary's Hospital, Imperial College Healthcare Trust

\section{INTRODUCTION}

- Hypothyroidism is a great mimicker of many common symptoms found at the emergency department.

- Myxoedema Coma is considered the most life threatening complication of hypothyroidism with a high mortality rate.

\section{CASE HISTORY}

- A 70 year old lady, with a background of known primary hypothyroidism presented to the Emergency department with a 1 day history of confusion and drowsiness.

- Her past medical history included rheumatoid arthritis.

- On presentation her vital signs included HR of 58bpm and temperature 28 degrees celsius.

- On examination, she had thin and brittle hair, loss of the lateral third of her eyebrows, and periorbital oedema

\section{INVESTIGATIONS}

- Blood results revealed an AKI with a creatinine of 213umol/L.

- ECG showed prolonged QTc with 1st degree heart block.

- Her troponin on admission was $1770 \mathrm{ng} / \mathrm{L}$, rising to $2190 \mathrm{ng} / \mathrm{L}$, and she was treated medically for an NSTEMI.

- Her thyroid function tests on admission showed TSH 236.98milliunit/L (0.30-4.20), free T4 6.1 pmol/L (9-23), free T3 2.7pmol/L (2.5-5.7).

- Thyroid peroxidase antibodies were strongly positive (646units/ml [0-75]).

- Short synacthen test was normal (0mins 342, 30mins 625, 60mins 693nmol/L).

- She was resuscitated with warm fluids, bear hugger and broad spectrum intravenous antibiotics.

\section{RESULTS}

- A diagnosis of myxoedema coma was made and she was treated with IV Liothyronine and oral thyroxine and IV hydrocortisone.

- Due to severe obtundation, she required ITU admission with ventilatory and inotropic support.

- The dose of liothyronine was carefully titrated, due to risk of causing further ischaemia in view of presentation with NSTEMI.

- She made a good recovery, with TFTs on discharge as follows: TSH 1.07milliunit/L, free T4 24.4pmol/L.

- She was discharged on oral Levothyroxine 175micrograms, with education on the importance of good medication adherence.

\section{CONCLUSIONS}

- Myxoedema is an important life threatening manifestation of hypothyroidism, which can result in fluid retention, negative inotropism and chronotropism with cardiogenic shock, stupor and coma.

- In severe cases overall mortality is $25-60 \%$.

- Prompt recognition and effective management of such patients is key to improving prognosis.

- It is also important to consider the need for steroids in stress doses, prior to administration of thyroid hormone replacement, when the possibility of coexisting adrenal insufficiency. 